Pacific Journal of Mathematics

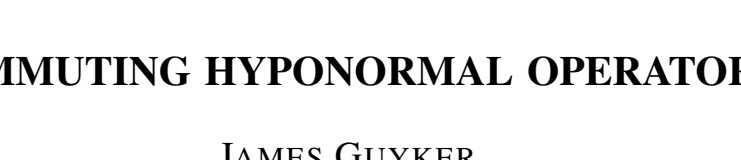




\title{
COMMUTING HYPONORMAL OPERATORS
}

\author{
JAMES GUYKER
}

\begin{abstract}
A hyponormal operator is normal if it commutes with a contraction $T$ of a Hilbert space, whose powers go to zero strongly, such that $1-T * T$ has finite-dimensional range and the coefficients of the characteristic function of $T$ lie in a commutative $C^{*}$-algebra. The hyponormal operator is a constant multiple of the identity transformation if the rank of $1-T * T$ is one.
\end{abstract}

Introduction. Let $T$ be a completely nonunitary contraction on Hilbert space such that $1-T^{*} T$ has closed range. There exists a power series $B(z)=\Sigma B_{n} z^{n}$ with operator coefficients which converges and is bounded by one in the unit disk such that $T$ is unitarily equivalent to the difference-quotient transformation in the de Branges-Rovnyak space $\mathscr{D}(B)$ [1, Theorem 4]. The characteristic function $B(z)$ is said to be of scalar type if $\left\{B_{n}: n \geqq 0\right\}$ is a commuting family of normal operators. Inner functions of scalar type were introduced and characterized in [10]. In this paper, it is shown that if $\left\{B_{n}: n=0, \cdots, N\right\}$ is a commuting family of normal operators, then polynomials $p(T)$ in $T$ of degree at most $N$ (weak limits of polynomials in $T$ if $B(z)$ is of scalar type) which satisfy $\|p(T) f\| \geqq$ $\left\|p(T)^{*} f\right\|$ for every $f$ in the range of $1-T^{*} T$ are restrictions of operators which commute with some completely nonunitary, partially isometric extension of $T$ and which satisfy a corresponding property. The construction is made in the space $\mathscr{D}\left(z^{M} B\right)$ for a given positive integer $M$, and is a modification of an extension procedure of de Branges [1, Theorem 9].

An operator $X$ on Hilbert space is called hyponormal if $\|X f\| \geqq$ $\left\|X^{*} f\right\|$ for every vector $f$. It is well-known [8] that if $X$ is a hyponormal contraction with no isometric part such that the rank of $1-X^{*} X$ is finite, then $X$ must be a normal operator acting on a finite-dimensional space. To ensure normality, the finite-rank hypothesis may not be replaced by a trace-class condition: for $0<p<\infty$, the weighted shift with weights $\left\{\left(1-\lambda_{n}\right)^{1 / 2}: n \geqq 0\right\}$ where $\left\{\lambda_{n}\right\}$ is a $p$-summable sequence of real numbers with the property that $0<$ $\lambda_{n} \leqq \lambda_{n-1} \leqq 1(n=1,2, \cdots)$ is a hyponormal, nonnormal contraction $X$ with no isometric part such that $1-X^{*} X$ is in the Schatten-von Neumann class $\mathscr{C}_{p}$.

A consequence of the above result in conjunction with the lifting theorems of Sarason [9] and Sz.-Nagy-Foias [11] is that if $T$ is a finite direct sum of $K$ contractions $T_{j}$, whose powers tend strongly 
to zero, such that the rank of $1-T_{j}^{*} T_{j}$ is one, and if $X$ is any operator which commutes with $T$ and satisfies $\|X f\| \geqq\left\|X^{*} f\right\|$ for all $f$ in the range of $1-T^{*} T$, then $X$ is normal with spectrum consisting of at most $K$ points. In particular, the only hyponormal operators commuting with the restriction of the backward shift to an invariant subspace are scalar multiples of the identity.

I am grateful to Professor Louis de Branges for several invaluable suggestions concerning this paper.

1. Preliminaries. For a fixed Hilbert space $\mathscr{C}$, the space $\mathscr{C}(z)$ is the Hilbert space of power series $f(z)=\Sigma a_{n} z^{n}$ with coefficients in $\mathscr{C}$ such that $\|\left. f(z)\right|^{2}=\Sigma\left|a_{n}\right|^{2}$ is finite. Let $B(z)=\Sigma B_{n} z^{n}$ be a power series whose coefficients are operators on $\mathscr{C}$, and suppose that for each fixed $z$ in the unit disk the series converges, in the strong operator topology, to an operator which is bounded by one. For $f(z)=\Sigma a_{n} z^{n}$ in $\mathscr{C}(z)$, the Cauchy product $B(z) f(z)=\Sigma\left(\sum_{k=0}^{n} B_{k} a_{n-k}\right) z^{n}$ is in $\mathscr{C}(z)$ and defines an operator bounded by one, which will be denoted by $T_{B}$, on $\mathscr{C}(z)$. The series $B(z)$ is an inner function if $T_{B}$ is a partial isometry.

The de Branges-Rovnyak space $\mathscr{H}(B)$ is the Hilbert space of series $f(z)$ in $\mathscr{C}(z)$ such that

$$
\|f(z)\|_{B}^{2}=\sup \left\{\|f(z)+B(z) g(z)\|^{2}-\|g(z)\|^{2}\right\}
$$

is finite, where the supremum is taken over all elements $g(z)$ of $\mathscr{C}(z)$ ([1], [2], [3]). The space $\mathscr{H}(B)$ is continuously embedded in $\mathscr{C}(z)$, and is isometrically embedded in $\mathscr{C}(z)$ if and only if $B(z)$ is inner, in which case $\mathscr{C}(z)=\mathscr{H}(B) \oplus$ (range $T_{B}$ ). If $f(z)$ is in $\mathscr{H}(B)$, then $(f(z)-f(0)) / z$ is in $\mathscr{C}(B)$ and $\left\|(f(z)-f(0)) / z||_{B}^{2} \leqq\right\| f(z) \|_{B}^{2}-|f(0)|^{2}$. The difference-quotient transformation

$$
R(0): f(z) \longrightarrow \frac{f(z)-f(0)}{z}
$$

defined on $\mathscr{H}(B)$ is a canonical model for contractions $T$ on Hilbert space with no isometric part (i.e., there is no nonzero vector $f$ such that $\left\|T^{n} f\right\|=\|f\|$ for every $\left.n=1,2, \cdots\right)$.

The operator $R(0)^{*}$ on $\mathscr{H}(B)$ is related to $R(0)$ on $\mathscr{H}\left(B^{*}\right)$ where $B^{*}(z)=\Sigma \bar{B}_{n} z^{n}$ if $B(z)=\Sigma B_{n} z^{n}$ and $\bar{B}_{n}$ is the adjoint of $B_{n}$ on $\mathscr{C}$. The space $\mathscr{D}(B)$ is the Hilbert space of pairs $(f(z), g(z))$ with $f(z)$ in $\mathscr{H}(B)$ and $g(z)$ in $\mathscr{C}\left(B^{*}\right)$ such that if $g(z)=\Sigma a_{n} z^{n}$ then

$$
z^{n} f(z)-B(z)\left(a_{0} z^{n-1}+\cdots+a_{n-1}\right)
$$

belongs to $\mathscr{H}(B)$ for every $n=1,2, \cdots$, and 
$\|(f(z), g(z))\|_{\mathscr{D}(B)}^{2}$

$$
=\sup \left\{\| z^{n} f(z)-B(z)\left(a_{0} z^{n-1}+\cdots+a_{n-1}\right)||_{B}^{2}+\left|a_{0}\right|^{2}+\cdots+\left|a_{n-1}\right|^{2}: n \geqq 1\right\}
$$

is finite. If $(f(z), g(z))$ is in $\mathscr{D}(B)$, then $\left(R(0) f(z), z g(z)-B^{*}(z) f(0)\right)$ is in $\mathscr{D}(B)$ and

$$
\left\|\left(R(0) f(z), z g(z)-B^{*}(z) f(0)\right)\right\|_{\mathscr{\mathscr { D }}(B)}^{2}=\|(f(z), g(z))\|_{\mathscr{Q}(B)}^{2}-|f(0)|^{2} .
$$

The difference-quotient transformation

$$
D:(f(z), g(z)) \longrightarrow\left(R(0) f(z), z g(z)-B^{*}(z) f(0)\right)
$$

defined on $\mathscr{D}(B)$ is a canonical model for completely nonunitary contractions $T$ on Hilbert space (i.e., there is no nonzero vector $f$ such that $\left\|T^{n} f\right\|=\|f\|=\left\|T^{*^{n}} f\right\|$ for every $\left.n=1,2, \cdots\right)$. The adjoint of $D$ is given by

$$
D^{*}:(f(z), g(z)) \longrightarrow(z f(z)-B(z) g(0), R(0) g(z))
$$

and satisfies $\left\|D^{*}(f(z), g(z))\right\|_{\mathscr{\mathscr { C }}(B)}^{2}=\|(f(z), g(z))\|_{\mathscr{O}(B)}^{2}-|g(0)|^{2}$ for every $(f(z), g(z))$ in $\mathscr{D}(B)$. If $D$ on $\mathscr{D}(B)$ has no isometric part, then $D$ is unitarily equivalent to $R(0)$ on $\mathscr{H}(B)$.

The space $\mathscr{D}(B)$ is a Hilbert space with a reproducing kernel function: for every $c$ in $\mathscr{C}$ and $w$ in the unit disk, the pairs

$$
\left(\frac{[1-B(z) \bar{B}(w)] c}{1-z \bar{w}}, \frac{\left[B^{*}(z)-\bar{B}(w)\right] c}{z-\bar{w}}\right)
$$

and

$$
\left(\frac{[B(z)-B(\bar{w})] c}{z-\bar{w}}, \frac{\left[1-B^{*}(z) B(\bar{w})\right] c}{1-z \bar{w}}\right)
$$

belong to $\mathscr{D}(B)$, where $\bar{B}(w)$ is the adjoint of $B(w)$ on $\mathscr{C}$, and if $(f(z), g(z))$ is an element of $\mathscr{D}(B)$, then

$\left\langle(f(z), g(z)), \quad\left(\frac{[1-B(z) \bar{B}(w)] c}{1-z \bar{w}}, \frac{\left[B^{*}(z)-\bar{B}(w)\right] c}{z-\bar{w}}\right)\right\rangle_{\mathscr{\mathscr { C }}(B)}=\langle f(w), c\rangle$

and

$$
\begin{gathered}
\left\langle(f(z), g(z)), \quad\left(\frac{[B(z)-B(\bar{w})] c}{z-\bar{w}}, \frac{\left[1-B^{*}(z) B(\bar{w})\right] c}{1-z \bar{w}}\right)\right\rangle_{\mathscr{D}(B)} \\
=\langle g(w), c\rangle .
\end{gathered}
$$

Suppose that $\mathscr{D}(A), \mathscr{D}(B)$ and $\mathscr{D}(C)$ are spaces such that $B(z)=A(z) C(z)$. If $(f(z), g(z))$ is in $\mathscr{D}(A)$ and if $(h(z), k(z))$ is in $\mathscr{D}(C)$, then

$$
(u(z), v(z))=\left(f(z)+A(z) h(z), C^{*}(z) g(z)+k(z)\right),
$$

is in $\mathscr{D}(B)$, and 


$$
\|(u(z), v(z))\|_{\mathscr{Q}(B)}^{2} \leqq\|(f(z), g(z))\|_{\mathscr{Q}(A)}^{2}+\|(h(z), k(z))\|_{\mathscr{D}(C)}^{2} .
$$

Moreover, every element $(u(z), v(z))$ in $\mathscr{D}(B)$ has a unique minimal decomposition in terms of $\mathscr{D}(A)$ and $\mathscr{D}(C)$ such that equality holds in the above inequality. Factorizations of $B(z)$ correspond to invariant subspaces of $D$.

2. The lifting theorem. In the following, $B(z)=\Sigma B_{n} z^{n}$ is a power series which converges and is bounded by one in the unit disk, where the coefficients are operators on a fixed Hilbert space $\mathscr{C}$.

LEMMA 1. If $B(z)=\Sigma B_{n} z^{n}$, and if $A$ is an operator on $\mathscr{C}$ which commutes with both $B_{n}$ and $\bar{B}_{n}$ for every $n$, then multiplication by $A$ is an operator on $\mathscr{D}(B)$, bounded by $\|A\|$, whose adjoint is multiplication by $\bar{A}$.

Proof. By [2, Theorem 4], the set of elements of the form $\left(1-T_{B} T_{B}^{*}\right) f(z)$, for $f(z)$ in $\mathscr{H}(B)$, is dense in $\mathscr{H}(B)$, and moreover

$$
\begin{aligned}
\left\|A\left(1-T_{B} T_{B}^{*}\right) f(z)\right\|_{B} & =\left\|\left(1-T_{B} T_{B}^{*}\right) A f(z)\right\|_{B} \\
& =\left\|\left(1-T_{B} T_{B}^{*}\right)^{1 / 2} A f(z)\right\| \\
& =\left\|A\left(1-T_{B} T_{B}^{*}\right)^{1 / 2} f(z)\right\| \\
& \leqq\|A\|\left\|\left(1-T_{B} T_{B}^{*}\right)^{1 / 2} f(z)\right\| \\
& =\|A\|\left\|\left(1-T_{B} T_{B}^{*}\right) f(z)\right\|_{B} .
\end{aligned}
$$

Multiplication by $A$ is therefore defined on a dense subspace of $\mathscr{H}(B)$ and has a continuous extension to all of $\mathscr{H}(B)$. Furthermore, since $\mathscr{H}(B)$ is continuously embedded in $\mathscr{C}(z)$, the extension coincides with the restriction of $T_{A}$ to $\mathscr{H}(B)$. Similarly, multiplication by $\bar{A}$ is an operator on $\mathscr{H}(B)$, and is the adjoint of multiplication by $A$ since for every $f(z)$ and $g(z)$ in $\mathscr{H}(B)$,

$$
\begin{aligned}
\left\langle A\left(1-T_{B} T_{B}^{*}\right) f(z), g(z)\right\rangle_{B} & =\left\langle\left(1-T_{B} T_{B}^{*}\right) A f(z), g(z)\right\rangle_{B} \\
& =\langle A f(z), g(z)\rangle \\
& =\langle f(z), \bar{A} g(z)\rangle \\
& =\left\langle\left(1-T_{B} T_{B}^{*}\right) f(z), \bar{A} g(z)\right\rangle_{B} .
\end{aligned}
$$

The lemma now follows from the definition of the norm in $\mathscr{D}(B)$ and the polarization identity.

The following result generalizes a direct consequence of Lemma 1. The convention $\sum_{r}^{s}(\cdot)=0$ when $s<r$ is observed.

LEMma 2. Let $B(z)=\Sigma B_{n} z^{n}$ and let $A$ be an operator on $\mathscr{C}$ which commutes with both $B_{n}$ and $\bar{B}_{n}$ for every $n=0, \cdots, N$. If $X$ and $Y$ (or $X^{*}$ and $Y^{*}$ ) are polynomials in the difference-quotient 
transformation $D$ in $\mathscr{D}(B)$ of degrees at most $N$ whose coefficients and their adjoints commute with $A$ and $B_{n}$ for every $n$, then

$$
\begin{aligned}
& \left\langle X\left([1-B(z) \bar{B}(0)] c, \frac{\left[B^{*}(z)-\bar{B}(0)\right] c}{z}\right),\right. \\
& \left.Y\left([1-B(z) \bar{B}(0)] A d, \frac{\left[B^{*}(z)-\bar{B}(0)\right] A d}{z}\right)\right\rangle_{\mathscr{D}(B)} \\
& \quad=\left\langle X\left([1-B(z) \bar{B}(0)] \bar{A} c, \frac{\left[B^{*}(z)-\bar{B}(0)\right] \bar{A} c}{z}\right),\right. \\
& \left.Y\left([1-B(z) \bar{B}(0)] d, \frac{\left[B^{*}(z)-\bar{B}(0)\right] d}{z}\right)\right\rangle_{\mathscr{S}(B)}
\end{aligned}
$$

for every $c$ and $d$ in $\mathscr{C}$.

Proof. Let $X=\sum_{0}^{N} A_{n} D^{n}$ and $Y=\sum_{0}^{N} C_{n} D^{n}$. Let the $n$th coefficient of the power series for $1-B(z) \bar{B}(0)$ be denoted by $\hat{B}_{n}$, and let $K(0, z) c=\left([1-B(z) \bar{B}(0)] c,\left(\left[B^{*}(z)-\bar{B}(0)\right] c\right) / z\right)$ for every $c$ in $\mathscr{C}$. By Lemma 1 , multiplication by $A_{n}$ and by $C_{n}$ are operators on $\mathscr{D}(B)$ for every $n$, and by the difference-quotient and polarization identities we have the following:

$$
\begin{aligned}
\left\langle A_{m+n}\right. & \left.D^{m+n} K(0, z) c, C_{n} D^{n} K(0, z) A d\right\rangle_{2(B)} \\
= & \left\langle D^{n} A_{m+n} D^{m} K(0, z) c, D^{n} K(0, z) C_{n} A d\right\rangle_{z(B) \mathscr{O}(B)} \\
= & \left\langle A_{m+n} D^{m} K(0, z) c, K(0, z) C_{n} A d\right\rangle_{\approx(B)} \\
& -\sum_{i=0}^{n-1}\left\langle A_{m+n} \hat{B}_{m+i} c, \hat{B}_{\imath} C_{n} A d\right\rangle \\
= & \left\langle A_{m+n} \hat{B}_{m} c, C_{n} A d\right\rangle-\sum_{i=0}^{n-1}\left\langle A_{m+n} \hat{B}_{m+i} \bar{A} c, \hat{B}_{i} C_{n} d\right\rangle \\
= & \left\langle A_{m+n} \hat{B}_{m} \bar{A} c, C_{n} d\right\rangle-\sum_{i=0}^{n-1}\left\langle A_{m+n} \hat{B}_{m+i} \bar{A} c, \hat{B}_{i} C_{n} d\right\rangle \\
= & \left\langle A_{m+n} D^{m+n} K(0, z) \bar{A} c, C_{n} D^{n} K(0, z) d\right\rangle_{\mathscr{D}}(B) .
\end{aligned}
$$

The identity now follows for $X$ and $Y$ by linearity and conjugation of inner products.

Similarly, the identity holds for $X^{*}$ and $Y^{*}$ polynomials in $D$ since

$$
\begin{aligned}
\left\langle D^{* m+n}\right. & \left.\bar{A}_{m+n} K(0, z) c, D^{* n} \bar{C}_{n} K(0, z) A d\right\rangle_{\mathscr{O}(B)} \\
= & \left\langle D^{* m} \bar{A}_{m+n} K(0, z) c, \bar{C}_{n} K(0, z) A d\right\rangle_{\mathscr{O}(B)} \\
& \quad-\sum_{i=1}^{n}\left\langle\bar{A}_{m+n} \bar{B}_{m+i} c, \bar{C}_{n} \bar{B}_{i} A d\right\rangle \\
= & \left\langle\bar{A}_{m+n} K(0, z) c, \bar{C}_{n} D^{m} K(0, z) A d\right\rangle_{\mathscr{O}(B)} \\
& -\sum_{i=1}^{n}\left\langle\bar{A}_{m+n} \bar{B}_{m+i} \bar{A} c, \bar{C}_{n} \bar{B}_{i} d\right\rangle
\end{aligned}
$$




$$
\begin{aligned}
= & \left\langle\bar{A}_{m+n} K(0, z) \bar{A} c, \bar{C}_{n} D^{m} K(0, z) d\right\rangle_{\mathscr{D}(B)} \\
& -\sum_{i=1}^{n}\left\langle\bar{A}_{m+n} \bar{B}_{m+i} \bar{A} c, \bar{C}_{n} \bar{B}_{i} d\right\rangle \\
= & \left\langle D^{* m+n} \bar{A}_{m+n} K(0, z) \bar{A} c, D^{*^{n}} \bar{C}_{n} K(0, z) d\right\rangle_{\mathscr{D}(B)} .
\end{aligned}
$$

Lemma 3. If $B(z)=\Sigma B_{n} z^{n}$ where $B_{i} \bar{B}_{j}=\bar{B}_{j} B_{i}$ for every $i, j=$ $0, \cdots, N$, and if $X$ is a polynomial of scalar type in the differencequotient transformation $D$ in $\mathscr{D}(B)$ of degree at most $N$ whose coefficients commute with $B_{n}$ for every $n$, then the following identity holds for every $c$ in $\mathscr{C}$ :

$$
\begin{aligned}
\| D X & \left([1-B(z) \bar{B}(0)] c, \frac{\left[B^{*}(z)-\bar{B}(0)\right] \underline{c}}{z}\right) \|_{\mathscr{Q}(B)}^{2} \\
& +\left\|X^{*}\left([1-B(z) \bar{B}(0)] \bar{B}(0) B(0) c, \frac{\left[B^{*}(z)-\bar{B}(0)\right] \bar{B}(0) B(0) c}{z}\right)\right\|_{\mathscr{Y}(B)}^{2} \\
= & \left\|D X^{*}\left([1-B(z) \bar{B}(0)] \bar{B}(0) c, \frac{\left[B^{*}(z)-\bar{B}(0)\right] \bar{B}(0) c}{z}\right)\right\|_{\mathscr{D}(B)}^{2} \\
& +\left\|X\left([1-B(z) \bar{B}(0)] B(0) c, \frac{\left[B^{*}(z)-\bar{B}(0)\right] B(0) c}{z}\right)\right\|_{\mathscr{B}(B)}^{2} .
\end{aligned}
$$

Proof. Let $X=\sum_{0}^{N} A_{n} D^{n}$, and let $\hat{B}_{n}$ and $K(0, z) c$ be defined as in Lemma 2. Let $\mathscr{F}$ be the family of transformations $T$ in $\mathscr{D}(B)$ which satisfy

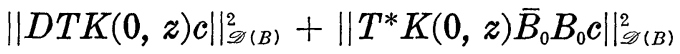

$$
\begin{aligned}
& =\left\|D T^{*} K(0, z) \bar{B}_{0} c\right\|_{\mathscr{D}(B)}^{2}+\left\|T K(0, z) B_{0} c\right\|_{\mathscr{Q}(B)}^{2}
\end{aligned}
$$

for every $c$ in $\mathscr{C}$.

By Fuglede's theorem [4], $A_{n}$ commutes with $\bar{B}_{m}$ for every $m$, and hence by Lemma 1 , multiplication by $A_{n}$ is a normal operator on $\mathscr{D}(B)$. Moreover, $A_{n} D^{n}$ is in $\mathscr{F}$ for every $n=0, \cdots, N$, since

$$
\begin{aligned}
& \left\|D\left(A_{n} D^{n}\right) K(0, z) c\right\|_{\mathscr{D}(B)}^{2}+\left\|D^{* n} \bar{A}_{n} K(0, z) \bar{B}_{0} B_{0} c\right\|_{\mathscr{D}(B)}^{2} \\
& =\left[\left\|K(0, z) A_{n} c\right\|_{\mathscr{Q}(B)}^{2}-\sum_{i=0}^{n}\left|\hat{B}_{i} A_{n} c\right|^{2}\right] \\
& +\left[\left\|K(0, z) \bar{B}_{0} B_{0} A_{n} c\right\|_{\mathscr{\mathscr { C }}(B)}^{2}-\sum_{i=1}^{n}\left|\overline{\widehat{B}}_{i} \bar{B}_{0} A_{n} c\right|^{2}\right] \\
& =\left(\left|A_{n} c\right|^{2}-\left|\bar{B}_{0} A_{n} c\right|^{2}\right)-\left(\left|A_{n} c\right|^{2}-2\left|\bar{B}_{0} A_{n} c\right|^{2}+\left|B_{0} \bar{B}_{0} A_{n} c\right|^{2}\right) \\
& +\left(\left|\bar{B}_{0} B_{0} A_{n} c\right|^{2}-\left|\bar{B}_{0}^{2} B_{0} A_{n} c\right|^{2}\right)-\sum_{i=1}^{n}\left(\left|\hat{B}_{i} A_{n} c\right|^{2}+\left|\hat{B}_{i} B_{0} A_{n} c\right|^{2}\right) \\
& =\left|B_{0} A_{n} c\right|^{2}-\left|B_{0}^{3} A_{n} c\right|^{2}-\sum_{i=1}^{n}\left(\left|\hat{B}_{i} A_{n} c\right|^{2}+\left|\hat{B}_{i} B_{0} A_{n} c\right|^{2}\right)
\end{aligned}
$$

and similarly

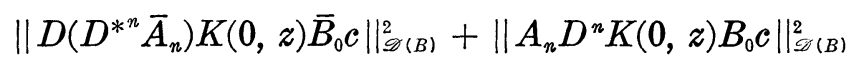




$$
\begin{aligned}
= & {\left[\left\|D^{* n} K(0, z) \bar{B}_{0} A_{n} c\right\|_{\mathscr{D}(B)}^{2}-\left|\overline{\widehat{B}}_{n} \bar{B}_{0} A_{n} c\right|^{2}\right] } \\
& \quad+\left[\left\|K(0, z) B_{0} A_{n} c\right\|_{\mathscr{D}(B)}^{2}-\sum_{i=0}^{n-1}\left|\hat{B}_{i} B_{0} A_{n} c\right|^{2}\right] \\
= & \left(\left|\bar{B}_{0} A_{n} c\right|^{2}-\left|\bar{B}_{0}^{2} A_{n} c\right|^{2}-\sum_{i=1}^{n}\left|\hat{B}_{i} A_{n} c\right|^{2}\right)+\left(\left|B_{0} A_{n} c\right|^{2}-\left|\bar{B}_{0} B_{0} A_{n} c\right|^{2}\right) \\
& \quad-\left(\left|B_{0} A_{n} c\right|^{2}-2\left|\bar{B}_{0} B_{0} A_{n} c\right|^{2}+\left|B_{0} \bar{B}_{0} B_{0} A_{n} c\right|^{2}\right)-\sum_{i=1}^{n}\left|\hat{B}_{i} B_{0} A_{n} c\right|^{2} \\
& \quad\left|B_{0} A_{n} c\right|^{2}-\left|B_{0}^{3} A_{n} c\right|^{2}-\sum_{i=1}^{n}\left(\left|\hat{B}_{i} A_{n} c\right|^{2}+\left|\hat{B}_{i} B_{0} A_{n} c\right|^{2}\right) .
\end{aligned}
$$

Next, observe that if $S$ and $T$ belong to $\mathscr{F}$, then $S+T$ belongs to $\mathscr{F}$ if and only if

$$
\operatorname{Re}\left[\left\langle T K(0, z) B_{0} c, S K(0, z) B_{0} c\right\rangle_{\mathscr{E}(B)}\right.
$$

$$
\begin{gathered}
\left.-\langle D T K(0, z) c, D S K(0, z) c\rangle_{\mathscr{O}(B)}\right] \\
=\operatorname{Re}\left[\left\langle T^{*} K(0, z) \bar{B}_{0} B_{0} c, S^{*} K(0, z) \bar{B}_{0} B_{0} c\right\rangle_{\mathscr{D}(B)}\right. \\
\left.-\left\langle D T^{*} K(0, z) \bar{B}_{0} c, D S^{*} K(0, z) \bar{B}_{0} c\right\rangle_{\mathscr{E}(B)}\right]
\end{gathered}
$$

for every $c$ in $\mathscr{C}$. For $m \geqq 1$, let $S=A_{n} D^{n}$ and $T=A_{m+n} D^{m+n}$. By the difference-quotient identity and polarization,

$$
\begin{gathered}
\left\langle T K(0, z) B_{0} c, S K(0, z) B_{0} c\right\rangle_{\mathscr{T}(B)}-\langle D T K(0, z) c, D S K(0, z) c\rangle_{\mathscr{D}(B)} \\
=\left\langle D^{n} D^{m} A_{m+n} K(0, z) B_{0} c, D^{n} K(0, z) A_{n} B_{0} c\right\rangle_{\mathscr{T}(B)} \\
-\left\langle D^{n} D^{m+1} A_{m+n} K(0, z) c, D^{n} D A_{n} K(0, z) c\right\rangle_{\mathscr{D}(B)} \\
=\left[\left\langle D^{m} A_{m+n} K(0, z) B_{0} c, K(0, z) A_{n} B_{0} c\right\rangle_{\mathscr{T}(B)}-\sum_{i=0}^{n-1}\left\langle A_{m+n} \hat{B}_{m+i} B_{0} c, \hat{B}_{i} A_{n} B_{0} c\right\rangle\right] \\
\quad-\left[\left\langle D D^{m} A_{m+n} K(0, z) c, D K(0, z) A_{n} c\right\rangle_{\mathscr{T}(B)}-\sum_{i=1}^{n}\left\langle A_{m+n} \hat{B}_{m+i} c, \hat{B}_{i} A_{n} c\right\rangle\right] \\
=\left[\left\langle A_{m+n} \hat{B}_{m} B_{0} c, A_{n} B_{0} c\right\rangle-\left\langle A_{m+n} \hat{B}_{m} c, A_{n} c\right\rangle+\left\langle A_{m+n} \hat{B}_{m} c, \hat{B}_{0} A_{n} c\right\rangle\right] \\
\quad+\sum_{i=1}^{n}\left\langle A_{m+n} \hat{B}_{m+i} c, A_{n} \hat{B}_{i} c\right\rangle-\sum_{i=0}^{n-1}\left\langle A_{m+n} \hat{B}_{m+i} B_{0} c, A_{n} \hat{B}_{i} B_{0} c\right\rangle \\
=\sum_{i=1}^{n}\left\langle A_{m+n} \bar{A}_{n} \hat{B}_{m+i} \overline{\hat{B}}_{i} c, c\right\rangle-\sum_{i=0}^{n-1}\left\langle\left(A_{m+n} \bar{A}_{n} \hat{B}_{m+i} \overline{\hat{B}}_{i}\right) B_{0} c, B_{0} c\right\rangle .
\end{gathered}
$$

Similarly,

$$
\begin{aligned}
& \left\langle T^{*} K(0, z) \bar{B}_{0} B_{0} c, S^{*} K(0, z) \bar{B}_{0} B_{0} c\right\rangle_{\mathscr{O}(B)} \\
& -\left\langle D T^{*} K(0, z) \bar{B}_{0} c, D S^{*} K(0, z) \bar{B}_{0} c\right\rangle_{\mathscr{D}(B)} \\
& =\left\langle D^{* n} D^{* m} \bar{A}_{m+n} K(0, z) \bar{B}_{0} B_{0} c, D^{* n} K(0, z) \bar{A}_{n} \bar{B}_{0} B_{0} c\right\rangle_{\mathscr{n}(B)} \\
& -\left\langle D^{* n} D^{*^{m}} \bar{A}_{m+n} K(0, z) \bar{B}_{0} c, D^{* n} K(0, z) \bar{A}_{n} \bar{B}_{0} c\right\rangle_{\mathscr{P}(B)} \\
& \quad+\left\langle\bar{A}_{m+n} \overline{\hat{B}}_{m+n} \bar{B}_{0} c, \bar{A}_{n} \overline{\hat{B}}_{n} \bar{B}_{0} c\right\rangle \\
& =\left[\left\langle D^{* m} \bar{A}_{m+n} K(0, z) \bar{B}_{0} B_{0} c, K(0, z) \bar{A}_{n} \bar{B}_{0} B_{0} c\right\rangle_{\mathscr{D}(B)}\right. \\
& \left.-\sum_{i=1}^{n}\left\langle\bar{A}_{m+n} \overline{\hat{B}}_{m+i} \bar{B}_{0} c, \bar{A}_{n} \overline{\hat{B}}_{i} \bar{B}_{0} c\right\rangle\right]
\end{aligned}
$$




$$
\begin{aligned}
-\left[\left\langle D^{* m} \bar{A}_{m+n} K(0, z) \bar{B}_{0} c, K(0, z) \bar{A}_{n} \bar{B}_{0} c\right\rangle_{\mathscr{O}(B)}-\sum_{i=1}^{n}\left\langle\bar{A}_{m+n} \overline{\hat{B}}_{m+i} c, \bar{A}_{n} \overline{\hat{B}}_{\imath} c\right\rangle\right] \\
\quad+\left\langle\bar{A}_{m+n} \overline{\hat{B}}_{m+n} \bar{B}_{0} c, \bar{A}_{n} \overline{\hat{B}}_{n} \bar{B}_{0} c\right\rangle \\
=\left[\left\langle\bar{A}_{m+n} \overline{\hat{B}}_{m} \bar{B}_{0} B_{0} c, \bar{A}_{n} \bar{B}_{0} B_{0} c\right\rangle-\left\langle\bar{A}_{m+n} \overline{\hat{B}}_{m} \bar{B}_{0} c, \bar{A}_{n} \bar{B}_{0} c\right\rangle\right] \\
\quad+\sum_{i=1}^{n}\left\langle\bar{A}_{m+n} \overline{\hat{B}}_{m+i} c, \bar{A}_{n} \overline{\hat{B}}_{i} c\right\rangle-\sum_{i=1}^{n-1}\left\langle\bar{A}_{m+n} \overline{\hat{B}}_{m+i} \bar{B}_{0} c, \bar{A}_{n} \overline{\hat{B}}_{i} \bar{B}_{0} c\right\rangle \\
=\sum_{i=1}^{n}\left\langle c, A_{m+n} \bar{A}_{n} \hat{B}_{m+i} \overline{\hat{B}}_{i} c\right\rangle-\sum_{i=0}^{n-1}\left\langle B_{0} c,\left(A_{m+n} \bar{A}_{n} \hat{B}_{m+i} \overline{\hat{B}}_{i}\right) B_{0} c\right\rangle .
\end{aligned}
$$

Taking real parts, we have that $\mathscr{F}$ contains $A_{n} D^{n}+A_{m+n} D^{m+n}$, and hence by the linearity of the inner products in (2.1), $\mathscr{F}$ contains $X$.

LEMma 4. If $B(z)$ is of scalar type, then the identity in Lemma 3 holds for weak limits $X$ of sequences of polynomials in the difference-quotient transformation $D$ whose coefficients lie in a (fixed) commutative $C^{*}$-algebra containing the coefficients of $B(z)$.

Proof. As in the proof of Lemma 3, the identity (2.1) holds whenever $S$ and $T$ are polynomials of scalar type in $D$ whose coefficients commute with the coefficients of $B(z)$. It follows that (2.1) holds for $S$ an arbitrary such polynomial in $D$ and $T=X$, and subsequently for $S=T=X$. Therefore $X$ satisfies the identity of Lemma 3.

REMARK 1. By Lemma 4 and Sarason's theorem [9], if the coefficient space $\mathscr{C}$ is one-dimensional and $B(z)$ is inner, then the identity in Lemma 3 holds for arbitrary operators $X$ commuting with $D$. This is false for spaces $\mathscr{C}$ of higher dimension, as the following example shows.

EXAMPLE. Let $B(z)=\left(\begin{array}{cc}b(z) & 0 \\ 0 & b(z)\end{array}\right)$ where $b(z)=\Sigma b_{n} z^{n}$ is a scalar inner function, and let $X=\left(\begin{array}{ll}0 & 0 \\ 1 & 0\end{array}\right) D$. Then the identity in Lemma 3 holds for $c=\left(\begin{array}{l}1 \\ 0\end{array}\right)$ only if either $b_{0}=0$ or $\left|b_{1}\right|=1-\left|b_{0}\right|^{2}$.

THEOREM 1. Let $D$ be the difference-quotient transformation in a space $\mathscr{D}(B)$, and suppose that $1-D^{*} D$ has closed range. Let $X$ be an operator on $\mathscr{D}(B)$ which satisfies

$$
\|X(f(z), g(z))\|_{\mathscr{D}(B)} \geqq\left\|X^{*}(f(z), g(z))\right\|_{\mathscr{D}(B)}
$$

for every $(f(z), g(z))$ in the range of $1-D^{*} D$. If $B(z)=\Sigma B_{n} z^{n}$ where either $B_{i} \bar{B}_{j}=\bar{B}_{j} B_{i}$ for every $i, j=0, \cdots, N$ and $X$ is a polynomial of scalar type in $D$ of degree at most $N$ whose coefficients 
commute with $B_{n}$ for every $n$, or, $B(z)$ is of scalar type and $X$ is the limit, in the weak operator topology, of a sequence of polynomials in $D$ whose coefficients lie in a commutative $C^{*}$-algebra containing $B_{n}$ for every $n$, then $X$ is unitarily equivalent to the restriction to an invariant subspace of an operator $Y=Y_{M}$ on $\mathscr{D}\left(z^{n} B\right)(M=1,2, \cdots)$ which commutes with the partially isometric difference-quotient transformation $V=V_{M}$ in $\mathscr{D}\left(z^{M} B\right)$ and which satisfies

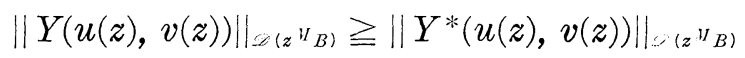

for every $(u(z), v(z))$ in the kernel of $V$. Moreover, $V=\left(\sum_{1}^{u} \oplus S_{j}^{*}\right) \oplus \hat{V}$ where $S_{j}$ is a truncated shift of index $j$ and the first $M$ powers of $\hat{V}$ are partial isometries such that the kernel of $\hat{V}^{*}$ has trivial intersection with the subspace $\sum_{1}^{M} \oplus \hat{V}^{* j-1}$ ker $\hat{V}$. If the dimension of $\mathscr{C}$ is finite, then $Y=\left(\sum_{1}^{M} \oplus Y_{j}\right) \oplus \hat{Y}$ where $Y_{j}$ and $\hat{Y}$ commute with $S_{j}^{*}$ and $\hat{V}$, respectively, and $Y_{j}$ is normal for every $j$. In this case, $Y=\left(\sum_{1}^{M} \oplus Z_{j}\right) \oplus Z$ where $Z_{j}$ is a normal operator on the space $V^{*^{j-1}} k e r V$, and $p(Y \ominus Z)=0$ for some nonzero (scalar) polynomial $p(z)$ of degree not exceeding the dimension of $\mathscr{C}$.

Proof. Since $\left\|\left(1-D D^{*}\right)^{1 / 2} D(f(z), g(z))\right\|_{\mathscr{D}(B)}=|\bar{B}(0) f(0)|$ for every $(f(z), g(z))$ in $\mathscr{D}(B)$ and $\left(1-D D^{*}\right)^{1 / 2} D=D\left(1-D^{*} D\right)^{1 / 2}$, with analogous identities for $1-D^{*} D$, it follows that the restriction of $1-D^{*} D$ to the closure of its range is unitarily equivalent to the restriction of $1-B_{0} \bar{B}_{0}$ to the closure of its range. Therefore, since $1-D^{*} D$ has closed range, so does $1-B_{0} \bar{B}_{0}$.

Let $K(0, z) c=\left([1-B(z) \bar{B}(0)] c,\left[B^{*}(z)-\bar{B}(0)\right] c / z\right)$ for every $c$ in $\mathscr{C}$. Define a transformation $\hat{\lambda}$ on $\mathscr{C}$ as follows: if $c=\left(1-B_{0} \bar{B}_{0}\right) d$ for some (uniquely determined) vector $d$ in the range of $1-B_{0} \bar{B}_{0}$, then $\hat{\lambda} c$ is the unique vector which satisfies

$$
\langle\hat{\lambda} c, a\rangle=\left\langle X K(0, z) B_{0} d, K(0, z) a\right\rangle_{\mathscr{D}(B)}
$$

for every $a$ in $\mathscr{C}$; if $\left(1-B_{0} \bar{B}_{0}\right) c=0$, define $\hat{\lambda} c$ to be the zero vector. Since $1-B_{0} \bar{B}_{0}$ has closed range, it follows that $\hat{\lambda}$ is continuous.

To compute $\hat{\lambda}^{*}$, observe that the range of $1-B_{0} \bar{B}_{0}$ reduces $\hat{\lambda}$ : let $b$ be in the kernel of $1-B_{0} \bar{B}_{0}$. Since $B_{0}$ is normal, $\left|\bar{B}_{0} b\right|=|b|=$ $\left|B_{0} b\right|$, and hence $\left(\left[B^{*}(z)-\bar{B}(0)\right] b\right) z=([B(z)-B(0)] b) z=0$. Moreover, the kernel of $1-B_{0} \bar{B}_{0}$ reduces $\bar{B}_{0}$, so that $K(0, z) b=(0,0)$. It follows that $b$ is orthogonal to $\hat{\lambda}\left(1-B_{0} \bar{B}_{0}\right) d$ for every vector $d$, and thus, since $b$ was arbitrary, the range of $1-B_{0} \bar{B}_{0}$ reduces $\hat{\lambda}$. Therefore, if $c=\left(1-B_{0} \bar{B}_{0}\right) d$ for some vector $d$ in the range of $1-B_{0} \bar{B}_{0}$, then by Lemmas 1 and $2, \hat{\lambda}^{*} c$ is the unique vector which satisfies

$$
\left\langle\widehat{\lambda}^{*} c, a\right\rangle=\left\langle X^{*} K(0, z) \bar{B}_{0} d, K(0, z) a\right\rangle_{\mathscr{\mathscr { C }}(B)}
$$


for every $a$ if $\mathscr{C}$; in $\left(1-B_{0} \bar{B}_{0}\right) c=0$, then clearly $\hat{\lambda}^{*} c=0$.

By the definitions of the norms in $\mathscr{H}(B)$ and $\mathscr{D}(B)$, it follows that the transformation

$$
W:(f(z), g(z)) \longrightarrow\left(z^{M} f(z), g(z)\right)
$$

takes $\mathscr{D}(B)$ isometrically into $\mathscr{D}\left(z^{M} B\right)$.

Let $(u(z), v(z))$ be in $\mathscr{D}\left(z^{M} B\right)$. The minimal decomposition of $(u(z), v(z))$ with respect to $\mathscr{D}(B)$ and $\mathscr{D}\left(z^{M}\right)$ is of the form

$$
(u(z), v(z))=\left(f(z)+B(z)\left(\sum_{0}^{M-1} c_{j} z^{j}\right), z^{M} g(z)+\sum_{0}^{M-1} c_{M-1-j} z^{j}\right)
$$

with $(f(z), g(z))$ in $\mathscr{D}(B)$ and $\left(\sum_{0}^{M-1} c_{j} z^{j}, \sum_{0}^{M-1} c_{M-1-j} z^{j}\right)$ in $\mathscr{D}\left(z^{M}\right)$ for some vectors $c_{j}$ in $\mathscr{C}$. Define a transformation $Y$ in $\mathscr{D}\left(z^{M} B\right)$ as follows:

$$
\begin{aligned}
& Y(u(z), v(z))=V^{M} W X(f(z), g(z)) \\
& \quad+\sum_{0}^{M-1} V^{j} W X\left(\frac{[B(z)-B(0)] c_{M-1-j}}{z},\left[1-B^{*}(z) B(0)\right] c_{M-1-j}\right) \\
& \quad+\left(\sum_{0}^{M-1}\left(\hat{\lambda} c_{j}\right) z^{j}, B^{*}(z)\left(\sum_{0}^{M-1}\left(\hat{\lambda} c_{M-1-j}\right) z^{j}\right)\right) .
\end{aligned}
$$

Since $V, W, X, \hat{\lambda}$, and minimal decompositions are linear, it follows that $Y$ is linear. Moreover, $Y$ is continuous since $V, W$, $X$, and $\hat{\lambda}$ are continuous and

$$
\|(u(z), v(z))\|_{\mathscr{D}\left(z^{M} B\right)}^{2}=\|(f(z), g(z))\|_{\mathscr{\mathscr { S }}(B)}^{2}+\sum_{0}^{M-1}\left|c_{j}\right|^{2} .
$$

By a straightforward computation,

$$
\begin{aligned}
& V Y(u(z), v(z))=V^{M} W D X(f(z), g(z)) \\
& \quad+\sum_{0}^{M-1} V^{j+1} W X\left(\frac{[B(z)-B(0)] c_{M-1-j}}{z},\left[1-B^{*}(z) B(0)\right] c_{M-1-j}\right) \\
& \quad+\left(\sum_{0}^{M-2}\left(\hat{\lambda} c_{j+1}\right) z^{j}, B^{*}(z)\left(\sum_{0}^{M-2}\left(\widehat{\lambda} c_{M-1-j}\right) z^{j+1}\right)\right) .
\end{aligned}
$$

Also by [1, Theorem 5(D)], the minimal decomposition of $V(u(z), v(z))$ in $\mathscr{D}\left(z^{M} B\right)$ is obtained with

$$
\left(f_{1}(z), g_{1}(z)\right)=D(f(z), g(z))+\left(\frac{[B(z)-B(0)] c_{0}}{z},\left[1-B^{*}(z) B(0)\right] c_{0}\right)
$$

in $\mathscr{D}(B)$ and

$$
\left(h_{1}(z), k_{1}(z)\right)=\left(\sum_{0}^{M-2} c_{j+1} z^{j}, \sum_{0}^{M-2} c_{M-1-j} z^{j+1}\right)
$$


in $\mathscr{D}\left(z^{M}\right)$. Therefore $\left.Y V(u z), v(z)\right)=V Y(u(z), v(z))$ since $X$ commutes with $D$.

Since

$$
\left(f(z), z^{M} g(z)\right)=\left(f(z)+B(z) \cdot 0, z^{M} g(z)+0\right)
$$

is minimal in $\mathscr{D}\left(z^{M} B\right)$ with $(f(z), g(z))$ in $\mathscr{D}(B)$ and $(0,0)$ in $\mathscr{D}\left(z^{M}\right)$, we have that $X$ is unitarily equivalent to the restriction of $Y$ to the subspace $V^{M} W \mathscr{D}(B)$.

The kernel of $V$ consists of all elements of the form $\left(c, z^{M-1} B^{*}(z) c\right)$ for $c$ in $\mathscr{C}$. The minimal decomposition of $\left(c, z^{M-1} B^{*}(z) c\right)$ in $\mathscr{D}\left(z^{M} B\right)$ is obtained with $K(0, z) c$ in $\mathscr{D}(B)$ and $\left(\bar{B}(0) c, z^{M-1} \bar{B}(0) c\right)$ in $\mathscr{D}\left(z^{M}\right)$. Therefore, since $V Y\left(c, z^{M-1} B^{*}(z) c\right)=Y V\left(c, z^{M-1} B^{*}(z) c\right)=(0,0)$, it follows that $Y\left(c, z^{M-1} B^{*}(z) c\right)=\left(d, z^{M-1} B^{*}(z) d\right)$ where $d$ is the unique vector which satisfies

$$
\langle d, a\rangle=\langle X K(0, z) c, K(0, z) a\rangle_{\mathscr{D}(B)}+\langle\hat{\lambda} \bar{B}(0) c, a\rangle
$$

for every $a$ in $\mathscr{C}$.

To compute the action of $Y^{*}$ on $\left(c, z^{M-1} B^{*}(z) c\right)$, let $(u(z), v(z))$ be in $\mathscr{D}\left(z^{M} B\right)$ and write

$$
(u(z), v(z))=\left(f(z)+B(z)\left(\sum_{0}^{M-1} c_{j} z^{j}\right), z^{M} g(z)+\sum_{0}^{M-1} c_{M-1-j} z^{j}\right)
$$

minimally with $(f(z), g(z))$ in $\mathscr{D}(B)$ and $\left(\sum_{0}^{M-1} c_{j} z^{j}, \sum_{1}^{M-1} c_{M-1-j} z^{j}\right)$ in $\mathscr{D}\left(z^{M}\right)$. Then

$$
\begin{aligned}
&\left\langle Y^{*}\left(c, z^{M-1} B^{*}(z) c\right),(u(z), v(z))\right\rangle_{\mathscr{D}\left(z^{M} B\right)} \\
& \quad=\langle K(0, z) c, X(f(z), g(z))\rangle_{\mathscr{D}(B)}+\left\langle c, \hat{\lambda} c_{0}\right\rangle \\
& \quad=\left\langle\left(f_{2}(z)+B(z) \hat{\lambda}^{*} c, z^{M} g_{2}(z)+z^{M-1} \widehat{\lambda}^{*} c\right),(u(z), v(z))\right\rangle_{\mathscr{O}\left(z^{M} M^{M}\right)}
\end{aligned}
$$

where $\left(f_{2}(z), g_{2}(z)\right)=X^{*} K(0, z) c$. Since $(u(z), v(z))$ was arbitrary, it follows that

$$
Y^{*}\left(c, z^{M-1} B^{*}(z) c\right)=\left(f_{2}(z)+B(z) \hat{\lambda}^{*} c, z^{M} g_{2}(z)+z^{M-1} \widehat{\lambda}^{*} c\right) .
$$

Since

$$
\left\|Y^{*}\left(c, z^{M-1} B^{*}(z) c\right)\right\|_{\mathscr{D}\left(z^{M} B\right)}^{2} \leqq\left\|X^{*} K(0, z) c\right\|_{\mathscr{D}(B)}^{2}+\left|\hat{\lambda}^{*} c\right|^{2}
$$

and

$$
\left\|Y\left(c, z^{M-1} B^{*}(z) c\right)\right\|_{\mathscr{Q}\left(z^{U}{ }_{B}\right)}^{2}=\left\|\left(d, z^{M-1} B^{*}(z) d\right)\right\|_{\mathscr{E}\left(z^{M} B\right)}^{2}=|d|^{2},
$$

it is sufficient to show

$$
\left\|X^{*} K(0, z) c\right\|_{\mathscr{D}(B)}^{2} \leqq|d|^{2}-\left|\widehat{\lambda}^{*} c\right|^{2}
$$

for all $c$ in $\mathscr{C}$, where $d=d(c)$ is defined by (2.2).

Let $c$ be in $\mathscr{C}$. Write $c=\left(1-B_{0} \bar{B}_{0}\right) a+b$ where $a$ is in the 
range of $1-B_{0} \bar{B}_{0}$ and $\left(1-B_{0} \bar{B}_{0}\right) b=0$. As above, $\hat{\lambda}^{*} b=0=\hat{\lambda} \bar{B}(0) b$ and $K(0, z) b=(0,0)$. Thus, we may assume $b=0$, and $c=\left(1-B_{0} \bar{B}_{0}\right) a$. In this case, by Lemmas 1 and 2 , and the normality of $B_{0}$,

$$
\begin{aligned}
& \left\|X^{*} K(0, z) c\right\|_{\mathscr{E}(B)}^{2} \\
& =\left\langle X^{*} K(0, z)\left(1-B_{0} \bar{B}_{0}\right) a, X^{*} K(0, z)\left(1-B_{0} \bar{B}_{0}\right) a\right\rangle_{\mathscr{Z}(B)} \\
& =\left\langle X^{*} K(0, z) a, X^{*} K(0, z)\left(1-B_{0} \bar{B}_{0}\right) a\right\rangle_{\mathscr{I}(B)} \\
& -\left\langle X^{*} K(0, z) B_{0} \bar{B}_{0} a, X^{*} K(0, z) a\right\rangle_{\mathscr{C}(B)}+\left\|X^{*} K(0, z) B_{0} \bar{B}_{0} a\right\|_{\mathscr{E}(B)}^{2} \\
& =\left\|X^{*} K(0, z)\left(1-\bar{B}_{0} B_{0}\right)^{1 / 2} a\right\|_{\mathscr{V}(B)}^{2}-\left\|X^{*} K(0, z) \bar{B}_{0} a\right\|_{\mathscr{E}(B)}^{2} \\
& +\left\|X^{*} K(0, z) \bar{B}_{0} B_{0} a\right\|_{\mathscr{E}(B)}^{2} .
\end{aligned}
$$

Therefore by hypothesis and Lemmas 1 and 2,

$$
\begin{aligned}
& \left\|X^{*} K(0, z) c\right\|_{\mathscr{Q}(B)}^{2} \\
& \leqq\left\|X K(0, z)\left(1-\bar{B}_{0} B_{0}\right)^{1 / 2} a\right\|_{\mathscr{\mathscr { Z }}(B)}^{2}-\left\|X^{*} K(0, z) \bar{B}_{0} \alpha\right\|_{\mathscr{D}(B)}^{2} \\
& +\left\|X^{*} K(0, z) \bar{B}_{0} B_{0} a\right\|_{\mathscr{\mathscr { V }}(B)}^{2} \\
& =\|X K(0, z) a\|_{\mathscr{Q}(B)}^{2}-\left\|X K(0, z) B_{0} a\right\|_{\mathscr{Q}(B)}^{2}-\left\|X^{*} K(0, z) \bar{B}_{0} a\right\|_{\mathscr{C}(B)}^{2} \\
& +\left\|X^{*} K(0, z) \bar{B}_{0} B_{0} a\right\|_{\mathscr{Z}(B)}^{2} \\
& =\left[\|D X K(0, z) a\|_{\mathscr{Q}(B)}^{2}+|d|^{2}\right]-\left\|X K(0, z) B_{0} a\right\|_{\mathscr{V}(B)}^{2} \\
& -\left[|| D X^{*} K(0, z) \bar{B}_{0} a \|_{\mathscr{Z}(B)}^{2}+\left|\hat{\lambda}^{*} c\right|^{2}\right]+\left\|X^{*} K(0, z) \bar{B}_{0} B_{0} a\right\|_{\mathscr{E}(B)}^{2}
\end{aligned}
$$

since $a=c+B_{0} \bar{B}_{0} a$. Hence by Lemmas 3 and 4 ,

$$
\left\|X^{*} K(0, z) c\right\|_{\mathscr{् Z}(B)}^{2} \leqq|d|^{2}-\left|\hat{\lambda}^{*} c\right|^{2}
$$

and therefore

$$
\|Y(u(z), v(z))\|_{\mathscr{Z}\left(z^{\left.M_{B}\right)}\right.} \geqq\left\|Y^{*}(u(z), v(z))\right\|_{\mathscr{D}\left(z^{M_{B}}\right)}
$$

for every $(u(z), v(z))$ in the kernel of $V$.

By [6, Lemma 2.2], $V, \cdots, V^{M}$ are partial isometries and hence so are their adjoints. The form of $V$ then follows from a slight modification of [5, Theorem 4.1]. In particular, $S_{j}$ is the restriction of $V^{*}$ to the space $\mathscr{H}_{j}=v$ (span) $\left\{V^{i} \mathscr{C}_{j}: i=0, \cdots, j-1\right\}$ where $\mathscr{C}_{j}=\operatorname{ker} V^{*} \cap V^{*^{j-1}} \operatorname{ker} V(j=1, \cdots, M)$.

Suppose that $\mathscr{C}$ is finite-dimensional. Since $Y V=V Y$, the kernel of $V$ is invariant under $Y$, and since it is finite-dimensional, the restriction $Z_{1}$ of $Y$ to the kernel of $V$ has an eigenvector, say $\left(e_{1}(z), e_{2}(z)\right)$. Since

$$
\left\|Y\left(e_{1}(z), e_{2}(z)\right)\right\|_{\mathscr{\mathscr { Z }}\left(z^{M} B\right)} \geqq\left\|Y^{*}\left(e_{1}(z), e_{2}(z)\right)\right\|_{\mathscr{\mathscr { C }}\left(z^{M} B\right)}
$$

it follows that $\left(e_{1}(z), e_{2}(z)\right)$ is a reducing eigenvector for $Y$. By considering the restriction of $Y$ to $\operatorname{ker} V \ominus\left\{\left(e_{1}(z), e_{2}(z)\right)\right\}$ and proceeding by induction, we have that the kernel of $V$ reduces $Y$, and $Z_{1}$ is normal. If $\lambda_{1}, \cdots, \lambda_{K}$ are the eigenvalues of $Z_{1}$ repeated according 
to multiplicity, then $p\left(Z_{1}\right)=0$ where $p(z)=\prod_{1}^{K}\left(z-\lambda_{j}\right)$. Also note that $\mathscr{Y}_{1}=\operatorname{ker} V^{*} \cap \operatorname{ker} V$ is a finite-dimensional invariant subspace of the normal operator $Z_{1}^{*}=\left.Y^{*}\right|_{k \text { er } y}$ and hence $\mathscr{C}_{1}$ reduces $Y$, and the restriction $Y_{1}$ of $Y$ to $\mathscr{C}_{1}$ is normal.

For the induction step, assume that $\mathscr{C}_{j}, \mathscr{C}_{j}$, and $V^{* j-1} \operatorname{ker} V$ $(j=1, \cdots, J-1 ; 2 \leqq J \leqq M)$ reduce $Y$, and the restriction of $Y$ to each of these subspaces is normal. Since the range of $V^{*}$ reduces $Y$, if $(r(z), s(z))$ is in the range of $V^{*}$, then $Y(r(z), s(z))=$ $V^{*} V Y(r(z), s(z))=V^{*} Y V(r(z), s(z))$ and $Y^{*}(r(z), s(z))=V^{*} Y^{*} V(r(z)$, $s(z))$.

Let $(u(z), v(z))$ be in $V^{*^{J-1}} \operatorname{ker} V$. Since $Y^{*} Y=Y Y^{*}$ on the space $V^{*^{J-2}} \operatorname{ker} V, V Y=Y V$, and $V(u(z), v(z))$ is in $V^{* J-2} \operatorname{ker} V$, it follows that

$$
\begin{aligned}
Y^{*} Y(u(z), v(z)) & =V^{*} Y^{*}\left(V V^{*}\right) Y V(u(z), v(z)) \\
& =V^{*} Y^{*} Y V(u(z), v(z)) \\
& =V^{*} Y Y^{*} V(u(z), v(z)) \\
& =V^{*} Y\left[\left(1-V V^{*}\right)+V V^{*}\right] Y^{*} V(u(z), v(z)) .
\end{aligned}
$$

Now $\left(1-V V^{*}\right) Y^{*} V(u(z), v(z))$ belongs to $\left(1-V V^{*}\right) V^{* J-2}$ ker $V$ which in turn is contained in $\operatorname{ker} V^{*} \cap V^{*, J-2} \operatorname{ker} V=\mathscr{C}_{J-1}$. By the induction hypothesis, $\mathscr{C}_{j-1}$ reduces $Y$. Therefore,

$$
\begin{aligned}
Y^{*} Y(u(z), v(z)) & =V^{*} Y V V^{*} Y^{*} V(u(z), v(z)) \\
& =Y Y^{*}(u(z), v(z)) .
\end{aligned}
$$

It follows that

$$
\|Y(u(z), v(z))\|_{\varepsilon\left(z^{\left.U_{B}\right)}\right.}=\left\|Y^{*}(u(z), v(z))\right\|_{\Sigma_{\left(z^{\prime I} B\right)}}
$$

for all $(u(z), v(z))$ in $V^{*^{J-1}} \operatorname{ker} V$, and, since $V^{*^{J-1}} \operatorname{ker} V$ is a finitedimensional invariant subspace for $Y^{*}$, we have that $V^{* J-1}$ ker $V$ reduces $Y$ as above, and the restriction $Z_{J}$ of $Y$ to $V^{* J-1} \operatorname{ker} V$ is normal. Clearly, $\quad p\left(\sum_{1}^{J} \oplus Z_{j}\right)=0$ since $\bar{p}\left(Y^{*}\right)\left(V^{*^{j-1}} \operatorname{ker} V\right)=$ $V^{*^{J-1}} \bar{p}\left(Z_{1}^{*}\right) \operatorname{ker} V=\{0\}$ for every $j=1, \cdots, J$.

Next, $\mathscr{C}_{J}$ reduces $Y$ and $\left.Y\right|_{\mathscr{C}_{J}}$ is normal since $\mathscr{C}_{J}=\operatorname{ker} V^{*} \cap$ $V^{*^{J-1}}$ ker $V$ is a finite-dimensional invariant subspace of $Y^{*}$, and the restriction of $Y^{*}$ to $V^{*^{J-1}} \operatorname{ker} V$ is normal.

Finally, $\mathscr{H}_{J}$ reduces $Y$ and $\left.Y\right|_{\mathscr{C}_{J}}$ is normal since $V^{i} \mathscr{C}_{J}(i=0, \cdots$, $J-1)$ is a finite-dimensional invariant subspace of $Y$ which is contained in $V^{*^{J-i-1}} \operatorname{ker} V$, and the restriction of $Y$ to $V^{*^{J-i-1}} \operatorname{ker} V$ is normal.

CoRollary 1. Let $D$ be the difference-quotient transformation in a space $\mathscr{D}(B)$ with a finite-dimensional coefficient space $\mathscr{C}$, and suppose that $D$ has no isometric part. Let $X$ be an operator on 
$\mathscr{D}(B)$ which satisfies

$$
\|X(f(z), g(z))\|_{\mathscr{D}(B)} \geqq\left\|X^{*}(f(z), g(z))\right\|_{\mathscr{S}(B)}
$$

for every $(f(z), g(z))$ in the range of $1-D^{*} D$. If $B(z)=\sum B_{n} z^{n}$ where $B_{i} \bar{B}_{j}=\bar{B}_{j} B_{i}$ for every $i, j=0, \cdots, N$, and $X$ is a polynomial of scalar type in $D$ of degree at most $N$ whose coefficients commute with $B_{n}$ for every $n$, then either $X$ is multiplication by an operator on $\mathscr{C}$ or the dimension of $\mathscr{D}(B)$ is finite $\left[\leqq N \times(\operatorname{dim} \mathscr{C})^{2}\right]$. Moreover, if $B(z)$ is of scalar type, and $X$ is the limit, in the weak operator topology, of a sequence of polynomials in $D$ whose coefficients lie in a commutative $C^{*}$-algebra containing $B_{n}$ for every $n$, then $p(X)=0$ for some nonzero (scalar) polynomial $p(z)$ of degree not exceeding the dimension of $\mathscr{C}$.

Proof. Since $D$ has no isometric part, $B(z) c$ is in $\mathscr{H}(B)$ for some vector $c$ only if $c=0$, and by [2, Lemma 4], $\mathscr{C}(B)$ contains no nonzero element of the form $B(z) c$. Therefore by the minimal decomposition of an element of $\mathscr{D}(z B)$ in terms of $\mathscr{D}(B)$ and $\mathscr{D}(z)$, it follows that the difference-quotient transformation $V$ on $\mathscr{D}(z B)$ has no isometric part. Moreover, as in the proof of Theorem 1, since $1-B_{0} \bar{B}_{0}$ has closed range, so does $1-D^{*} D$.

By Theorem $1, X$ is unitarily equivalent to a part of an operator $Y$ on $\mathscr{D}(z B)$ which commutes with $V$ and satisfies

$$
\|Y(u(z), v(z))\|_{\mathscr{D}(z B)} \geqq\left\|Y^{*}(u(z), v(z))\right\|_{\mathscr{D}(z B)}
$$

for all $(u(z), v(z))$ in the kernel of $V$. Moreover, the kernel of $V$ reduces $Y$ and $p(Y)$ ker $V=\{0\}$ for some nonzero polynomial $p(z)$ of degree at most the dimension of $\mathscr{C}$. Since $V$ has no isometric part, $\mathscr{D}(z B)$ is the closed span of the subspaces $V^{* n} \operatorname{ker} V(n=0,1, \cdots)$. Therefore, since $\bar{p}\left(Y^{*}\right)$ commutes with $V^{*^{n}}, p(Y)=0$ and hence $P(X)=0$.

Suppose that $X$ is a nonconstant, scalar type polynomial in $D$ of degree at most $N$. By the above, $q(D)=0$ for some scalar type polynomial $q(z)$ of degree at most $N \times \operatorname{dim} \mathscr{C}$. Since $D$ has no isometric part, $D$ is unitarily equivalent to $R(0)$ on $\mathscr{H}(B)$. Since any countable family of commuting normal operators on a finite-dimensional space has a common eigenvector, $q(R(0))(=0)$ is the restriction of an operator on $\mathscr{C}(z)$ of the form $\sum_{1}^{\operatorname{dim} \mathscr{E}} \oplus q_{i}\left(R(0)_{i}\right)$ where $q_{i}(z)$ is a scalar polynomial of degree at most $N \times \operatorname{dim} \mathscr{C}$ and $R(0)_{i}$ is the difference-quotient transformation on $\mathscr{C}_{i}(z)$ where $\mathscr{C}_{i}$ is onedimensional. Since the eigenspace corresponding to an eigenvalue of $R(0)_{i}$ is one-dimensional, and since the dimension of the kernel of a finite product of operators does not exceed the sum of the dimensions of the kernels of the factors, it follows that the dimension of 
$\mathscr{H}(B)$ (and hence of $\mathscr{D}(B))$ does not exceed $N \times(\operatorname{dim} \mathscr{C})^{2}$.

3. Applications. The following result extends [3, Problem 110] and [7, Corollary 1].

THEOREM 2. Let $T$ be a contraction on Hilbert space such that rank $\left(1-T T^{*}\right) \leqq \operatorname{rank}\left(1-T^{*} T\right)=1$, and suppose that $T$ has no isometric part. If $X$ is the weak limit of a sequence of polynomials in $T$, and if $f$ is a nonzero vector in the range of $1-T^{*} T$, then $\|X f\| \leqq\left\|X^{*} f\right\|$ with equality holding only if $X$ is a scalar multiple of the identity.

Proof. By [2, Theorem 1] and [3, Theorem 15], $T$ is unitarily equivalent to the difference-quotient transformation in a space $\mathscr{D}(B)$ where the coefficient space is one-dimensional. The theorem now follows by applying Corollary 1 .

THEOREM 3. Let $T$ be a contraction on Hilbert space such that $T^{n}(n=1,2, \cdots)$ tends strongly to zero, and suppose that $T=$ $\sum_{1}^{K} \oplus T_{j}$ where the rank of $1-T_{j}^{*} T_{j}$ is one for every $j$. If $X$ is an operator which commutes with $T$ and satisfies $\|X f\| \geqq\left\|X^{*} f\right\|$ for every vector $f$ in the range of $1-T^{*} T$, then $X$ is normal with spectrum consisting of at most $K$ points.

Proof. By [3, Theorem 12], there exist scalar inner functions $b_{j}(z)(j=1, \cdots, K)$ such that $T$ is unitarily equivalent to the difference-quotient transformation $R(0)$ in $\mathscr{H}(B)$ where

$$
B(z)=\left(\begin{array}{cccc}
b_{1}(z) & & & 0 \\
& \cdot & \cdot & \\
0 & & & b_{K}(z)
\end{array}\right)
$$

is an inner function of scalar type. The proof proceeds by induction on $K$.

If $K=1$, then by Sarason's theorem [9], $X$ is the weak limit of a sequence of polynomials in $R(0)$; and hence by [3, Theorem 13] and Theorem 2, $X$ is a scalar multiple of the identity.

Assume that the theorem is true for the difference-quotient transformations in spaces $\mathscr{H}(B)$ of the form $\mathscr{H}(B)=\sum_{1}^{L} \oplus \mathscr{H}\left(b_{j}\right)$ for all integers $L, 1 \leqq L<K$, where the $b_{j}$ 's are scalar inner functions. Let $X$ commute with $R(0)$ on $\mathscr{H}(B)=\sum_{1}^{K} \oplus \mathscr{H}\left(b_{j}\right)$ and satisfy $\|X f(z)\| \geqq\left\|X^{*} f(z)\right\|$ for every $f(z)$ in the range of $1-R(0)^{*} R(0)$, where $b_{j}(z)$ is a scalar inner function for every $j$. By the Sz.-Nagy-Foias lifting theorem [11], $X$ is the restriction of 
an operator on $\mathscr{C}(z)=\sum_{1}^{K} \oplus \mathscr{C}_{j}(z)$ of the form $\left(T_{\varphi_{i i}}^{*}\right)_{K \times K}$ where $\mathscr{C}_{j}$ is the space of complex numbers and $\varphi_{i j}(z)$ is a bounded analytic (scalar) function on the unit disk for all $i$ and $j$. Moreover, since $\mathscr{H}(B)$ is invariant under $\left(T_{\varphi_{i j}}^{*}\right)_{K \times K}$, the range of $T_{B}$ is invariant under $\left(T_{\varphi_{j i}}\right)_{K \times K}$, and hence for each $k, 1 \leqq k \leqq K, \varphi_{j k}(z) b_{j}(z)$ is contained in the range of $T_{b_{k}}$ for every $j=1, \cdots, K$.

For a fixed integer $j_{0}\left(1 \leqq j_{0} \leqq K\right)$, consider an element of $\mathscr{C}(B)$ of the form $f(z)=\sum_{1}^{K} \oplus\left[1-b_{j}(z) \bar{b}_{j}(0)\right] x_{j}$ where $x_{j_{0}}=1$ and $x_{j}=0$ for all $j \neq j_{0}$. Since $f(z)$ is in the range of $1-R(0)^{*} R(0)$, we have that

$$
\begin{aligned}
\|X f(z)\|^{2} & =\sum_{i=1}^{K}\left\|T_{\zeta j_{0}}^{*}\left[1-b_{j_{0}}(z) \bar{b}_{j_{0}}(0)\right]\right\|^{2} \\
& \geqq\left\|X^{*} f(z)\right\|^{2} \\
& =\sum_{i=1}^{K}\left\|P_{i} T_{\varphi_{i_{0}}}\left[1-b_{j_{0}}(z) \bar{b}_{j_{0}}(0)\right]\right\|^{2} \\
& =\sum_{i=1}^{K}\left\|P_{i} \varphi_{j_{0} i}(z)\right\|^{2}
\end{aligned}
$$

where $P_{i}$ is the (orthogonal) projection of $\mathscr{C}_{i}(z)$ onto $\mathscr{C}\left(b_{i}\right)$. Moreover, by the case $K=1$,

$$
\begin{aligned}
\left\|P_{j_{0}} \varphi_{i j_{0}}(z)\right\| & =\left\|P_{j_{0}} T_{\varphi_{i j_{0}}}\left[1-b_{j_{0}}(z) \bar{b}_{j_{0}}(0)\right]\right\| \\
& \geqq\left\|T_{\varphi_{i j_{0}}}^{*}\left[1-b_{j_{0}}(z) \bar{b}_{j_{0}}(0)\right]\right\|
\end{aligned}
$$

for every $i=1, \cdots, K$. Therefore,

$$
\begin{aligned}
\sum_{\substack{i=1 \\
\imath \neq j_{0}}}^{K}\left\|P_{j_{0}} \Phi_{i j_{0}}(z)\right\|^{2} & \geqq \sum_{\substack{i=1 \\
i \neq j_{0}}}^{K}\left\|T_{\varphi_{i j_{0}}}^{*}\left[1-b_{j_{0}}(z) \bar{b}_{j_{0}}(0)\right]\right\|^{2} \\
& \geqq \sum_{\substack{i=1 \\
i \neq j_{0}}}^{K}\left\|P_{i} \varphi_{j_{0} i}(z)\right\|^{2}
\end{aligned}
$$

which holds for all $j_{0}=1, \cdots, K$.

Combining the above inequalities, by induction we have the following:

$$
\begin{aligned}
\sum_{i=2}^{K} \| & P_{1} \varphi_{i 1}(z) \|^{2} \\
& \geqq \sum_{i=2}^{K}\left\|T_{\varphi_{i 1}}^{*}\left[1-b_{1}(z) \bar{b}_{1}(0)\right]\right\|^{2} \\
& \geqq \sum_{i=2}^{K}\left\|P_{i} \varphi_{1 i}(z)\right\|^{2} \\
& \geqq \sum_{i=2}^{K}\left(\sum_{\substack{j=1 \\
j \neq \imath}}^{K}\left\|T_{\varphi_{i i}}^{*}\left[1-b_{i}(z) \bar{b}_{i}(0)\right]\right\|^{2}-\sum_{\substack{j=2 \\
j \neq i}}^{K}\left\|P_{i} \varphi_{j i}(z)\right\|^{2}\right)
\end{aligned}
$$




$$
\begin{aligned}
& \geqq \sum_{i=2}^{K}\left(\sum_{\substack{j=1 \\
j \neq i}}^{K}\left\|P_{j} \varphi_{i j}(z)\right\|^{2}-\sum_{\substack{j=2 \\
j \neq i}}^{K}\left\|P_{i} \varphi_{j i}(z)\right\|^{2}\right) \\
& =\sum_{i=2}^{K}\left\|P_{1} \varphi_{i 1}(z)\right\|^{2} .
\end{aligned}
$$

The above inequalities are therefore equalities and in particular

$$
\begin{aligned}
\sum_{\substack{j=1 \\
j \neq i}}^{K}\left\|P_{i} T_{\varphi_{j i}}\left[1-b_{i}(z) \bar{b}_{i}(0)\right]\right\|^{2} & =\sum_{\substack{j=1 \\
j \neq i}}^{K}\left\|T_{\varphi_{j i}}^{*}\left[1-b_{i}(z) \bar{b}_{i}(0)\right]\right\|^{2} \\
& =\sum_{\substack{j=1 \\
j \neq i}}^{K}\left\|P_{j} T_{\varphi_{i j}}\left[1-b_{i}(z) \bar{b}_{i}(0)\right]\right\|^{2} \\
& \leqq \sum_{\substack{j=1 \\
j \neq i}}^{K}\left\|T_{\varphi_{i j}}\left[1-b_{i}(z) \bar{b}_{i}(0)\right]\right\|^{2}
\end{aligned}
$$

for every $i=1, \cdots, K$. Hence by the case $K=1$, it follows that the restriction of $T_{\varphi_{j i}}^{*}$ to $\mathscr{C}\left(b_{i}\right)$ is a scalar $\lambda_{j i}$ times the identity for all $j \neq i$, and

$$
\sum_{\substack{j=1 \\ j \neq i}}^{K}\left|\lambda_{j i}\right|^{2} \leqq \sum_{\substack{j=1 \\ j \neq i}}^{K}\left|\lambda_{i j}\right|^{2}
$$

for every $i=1, \cdots, K$. Therefore by (3.1) and the case $K=1$, the restriction of $T_{\varphi_{i i}}^{*}$ to $\mathscr{H}\left(b_{i}\right)$ is a scalar $\lambda_{i i}$ times the identity for every $i=1, \cdots, K$, and consequently $X=\left(\lambda_{i j}\right)_{K \times K}$.

Suppose first that $\mathscr{H}\left(b_{i}\right)=\mathscr{H}\left(b_{j}\right)$ for all $i$ and $j$. In this case, the range of $1-R(0)^{*} R(0)$ reduces $X$, and since it is finite-dimensional and the restriction of $X$ to it is hyponormal, it follows that $X X^{*}=X^{*} X$ on the range of $1-R(0)^{*} R(0)$.

Let $h(z)$ be an arbitrary element of $\mathscr{H}(B)$. Then $h(z)$ is the limit of a sequence of vectors of the form $\sum_{0}^{n} R(0)^{* j} f_{j}(z)$ where $f_{j}(z)$ is in the range of $1-R(0)^{*} R(0)$ for every $j$. Since $X X^{*}$ and $X^{*} X$ commute with $R(0)^{* j}$, we have that $X X^{*} h(z)=X^{*} X h(z)$. Hence $X$ is normal.

Let $\lambda_{1}, \cdots, \lambda_{K}$ be the eigenvalues of the restriction of $X$ to the range of $1-R(0)^{*} R(0)$, listed according to multiplicity, and let $\eta_{j}=$ $\vee\left\{f(z) \in \mathscr{H}(B): X f(z)=\lambda_{j} f(z)\right\}$. Since $X$ is normal, if $\lambda_{i} \neq \lambda_{j}$, then $\eta_{i}$ is orthogonal to $\eta_{j}$. Moreover, since $R(0)$ has no isometric part and $X R(0)^{*}=R(0)^{*} X$, it follows that $\mathscr{H}(B)=\vee\left\{\eta_{j}: j=1, \cdots, K\right\}$. Therefore, $X$ is diagonalizable with $\operatorname{sp}(X)=\left\{\lambda_{j}: j=1, \cdots, K\right\}$.

Finally, suppose that $\mathscr{C}\left(b_{i}\right) \neq \mathscr{C}\left(b_{j}\right)$ for at least one pair $(i, j)$. There exists a space $\mathscr{C}\left(b_{i_{0}}\right)$ which is minimal in the sense that for every $i$ either $\mathscr{H}\left(b_{i}\right)=\mathscr{H}\left(b_{i_{0}}\right)$ or $\mathscr{C}\left(b_{i}\right)$ is not contained in $\mathscr{H}\left(b_{i_{0}}\right)$. Let $\Omega$ be the set of indices $i$ such that $\mathscr{H}\left(b_{i}\right)=\mathscr{H}\left(b_{i_{0}}\right)$. Then $\Omega \neq\{1, \cdots, K\}$ by assumption, and for every $i$ in $\Omega$ and $j$ not in $\Omega$, 
$\lambda_{i j}=0 . \quad$ By (3.2),

$$
\sum_{\substack{i \in \Omega \\ j \notin \Omega}}\left|\lambda_{j i}\right|^{2} \leqq \sum_{i, j \in \Omega}\left(\left|\lambda_{i j}\right|^{2}-\left|\lambda_{j i}\right|^{2}\right)=0
$$

Therefore, $\lambda_{i j}=0=\lambda_{j i}$ for every $i$ in $\Omega$ and $j$ not in $\Omega$. It follows that the space $\sum_{i \in \Omega} \oplus \mathscr{H}\left(b_{i}\right)$ reduces $X$, that the restriction of $X$ to this space satisfies the induction hypothesis and hence is normal with spectrum consisting of at most card $\Omega$ points. Similarly, the restriction of $X$ to $\sum_{i \notin \Omega} \oplus \mathscr{H}\left(b_{i}\right)$ is normal with spectrum at most $K$ - card $\Omega$ points, and consequently $X$ is normal with spectrum at most $K$ points.

CoRollary 2. Let $X$ commute with the difference-quotient transformation $D$ in a space $\mathscr{D}(B)$ where $B(z)$ is an inner function of scalar type and the coefficient space $\mathscr{C}$ is finite-dimensional. If

$$
\|X(f(z), g(z))\|_{\mathscr{D}(B)} \geqq\left\|X^{*}(f(z), g(z))\right\|_{\mathscr{D}(B)}
$$

for every $(f(z), g(z))$ in the range of $1-D^{*} D$, then $X$ is a normal operator whose spectrum consists of a finite number (ฏdim $\mathscr{C}$ ) of points.

Proof. Since any countable family of commuting normal operators on a finite-dimensional space has a common eigenvector, it follows that $\mathscr{D}(B)=\sum_{1}^{\operatorname{dim} \mathscr{G}} \oplus \mathscr{D}\left(b_{j}\right)$ where $b_{j}(z)$ is a scalar inner function for all $j$. Corollary 2 is therefore an immediate consequence of Theorem 3 .

REMARK 2. The analytic Toeplitz operator $T_{\varphi}$ on $\mathscr{C}(z)$ with $\mathscr{C}$ one-dimensional, for the symbol $\varphi(z)$ an inner function, is a universal model for unilateral shifts. Therefore, the restriction of $T_{\varphi}^{*}$ to an arbitrary invariant subspace is a canonical model for contractions whose powers tend strongly to zero. A consequence of Corollary 2 is that the restriction of $T_{\varphi}^{*}$ to an arbitrary invariant subspace of the backward shift $T_{z}^{*}$ is never hyponormal (i.e., only if it is a scalar times the identity).

\section{REFERENCES}

1. L. de Branges, Factorization and invariant subspaces, J. Math. Anal. Appl., 29 (1970), 163-200.

2. L. de Branges and J. Rovnyak, Canonical models in quantum scattering theory, Perturbation Theory and its Applications in Quantum Mechanics, pp. 295-391, Wiley, New York, 1966.

3. Square Summable Power Series, Holt, Rinehart, and Winston, New York, 1966. 
4. B. Fuglede, A commutativity theorem for normal operators, Proc. N. A. S., 36 (1950), $35-40$.

5. J. Guyker, A structure thearem for operators with closed range, Bull. Austral. Math. Soc., 18 (1978), 169-186.

6. - On partial isometries with no isometric part, Pacific J. Math., 62 (1976), 419-433.

7. - Reducing subspaces of contractions with no isometric part, Proc. Amer. Math. Soc., 45 (1974), 411-413.

8. C. R. Putnam, An inequality for the area of hyponormal spectra, Math.Z., 116 (1970), 323-330.

9. D. Sarason, Generalized interpolation in $H^{\infty}$, Trans. Amer. Math. Soc., 127 (1967), 179-203.

10. M. J. Sherman, A spectral theory for inner functions, Trans. Amer. Math. Soc., 135 (1969), 387-398.

11. B. Sz.-Nagy and C. Foiaș, Dilatation des commutants d'opérateurs, C. R. Acad. Sci. Paris, 266 (1968), 493-495.

Received June 5, 1979 and in revised form February 21, 1980.

SUNY College at Buffalo

Buffalo, N.Y. 14222

AND

Purdue University

LAFAyETte, IN 47907 



\section{PACIFIC JOURNAL OF MATHEMATICS}

\section{EDITORS}

DONALD BABBITT (Managing Editor)

University of California

Los Angeles, CA 90024

Hugo RossI

University of Utah

Salt Lake City, UT 84112

C. C. MOORE and ANDREW OGG

University of California

Berkeley, CA 94720

\section{J. DugundjI}

Department of Mathematics

University of Southern California

Los Angeles, CA 90007

R. FinN and J. Milgram

Stanford University

Stanford, CA 94305

\section{ASSOCIATE EDITORS}
E. F. BeCKENBACH
B. H. Neumann
F. WoLF
K. YoSHIDA

\section{SUPPORTING INSTITUTIONS}

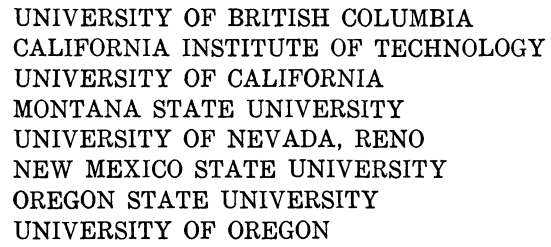

UNIVERSITY OF BRITISH COLUMBIA CALIFORNIA INSTITUTE OF TECHNOLOGY UNIVERSITY OF CALIFORNIA MONTANA STATE UNIVERSITY UNIVERSITY OF NEVADA, RENO NEW MEXICO STATE UNIVERSITY OREGON STATE UNIVERSITY UNIVERSITY OF OREGON

\author{
UNIVERSITY OF SOUTHERN CALIFORNIA \\ STANFORD UNIVERSITY \\ UNIVERSITY OF HAWAII \\ UNIVERSITY OF TOKYO \\ UNIVERSITY OF UTAH \\ WASHINGTON STATE UNIVERSITY \\ UNIVERSITY OF WASHINGTON
}

The Supporting Institutions listed above contribute to the cost of publication of this Journal, but they are not owners or publishers and have no responsibility for its content or policies.

Mathematical papers intended for publication in the Pacific Journal of Mathematics should be in typed form or offset-reproduced, (not dittoed), double spaced with large margins. Please do not use built up fractions in the text of the manuscript. However, you may use them in the displayed equations. Underline Greek letters in red, German in green, and script in blue. The first paragraph or two must be capable of being used separately as a synopsis of the entire paper. Please propose a heading for the odd numbered pages of less than 35 characters. Manuscripts, in triplicate, may be sent to any one of the editors. Please classify according to the scheme of Math. Reviews, Index to Vol. 39. Supply name and address of author to whom proofs should be sent. All other communications should be addressed to the managing editor, or Elaine Barth, University of California, Los Angeles, California, 90024.

50 reprints to each author are provided free for each article, only if page charges have been substantially paid. Additional copies may be obtained at cost in multiples of 50 .

The Pacific Journal of Mathematics is issued monthly as of January 1966. Regular subscription rate: $\$ 84.00$ a year (6 Vols., 12 issues). Special rate: $\$ 42.00$ a year to individual members of supporting institutions.

Subscriptions, orders for numbers issued in the last three calendar years, and changes of address shoud be sent to Pacific Journal of Mathematics, P.O. Box 969, Carmel Valley, CA 93924, U.S.A Old back numbers obtainable from Kraus Periodicals Co., Route 100, Millwood, NY 10546.

PUBLISHED BY PACIFIC JOURNAL OF MATHEMATICS, A NON-PROFIT CORPORATION

Printed at Kokusai Bunken Insatsusha (International Academic Printing Co., Ltd.). 8-8, 3-chome, Takadanobaba, Shinjuku-ku, Tokyo 160, Japan. 


\section{Pacific Journal of Mathematics}

\section{Vol. 91, No. 2 December, 1980}

Victor P. Camillo and Julius Martin Zelmanowitz, Dimension modules ... . . 249

Yonina S. Cooper, Stable sequences in pre-abelian categories ........... 263

Chandrakant Mahadeorao Deo and H. Ship-Fah Wong, On Berry-Esseen approximation and a functional LIL for a class of dependent random fields.........................................

H. P. Dikshit and S. N. Dubey, $|C, 1|$ summability of series associated with

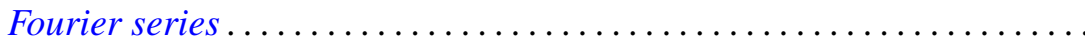

M. Edelstein, On the homomorphic and isomorphic embeddings of a semiflow into a radial flow.

Gilles Godefroy, Compacts de Rosenthal ..................... 293

James Guyker, Commuting hyponormal operators ................ 307

Thomas Eric Hall and Peter R. Jones, On the lattice of varieties of bands of

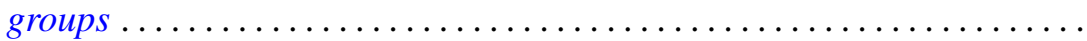

Taqdir Husain and Saleem H. Watson, Topological algebras with orthogonal Schauder bases ....................................

V. K. Jain, Some expansions involving basic hypergeometric functions of two variables. . .

Joe W. Jenkins, On group actions with nonzero fixed points ........... 363

Michael Ellsworth Mays, Groups of square-free order are scarce ........ 373

Michael John McAsey, Canonical models for invariant subspaces... 377

Peter A. McCoy, Singularities of solutions to linear second order elliptic partial differential equations with analytic coefficients by approximation methods...

Terrence Millar, Homogeneous models and decidability.

Stephen Carl Milne, A multiple series transformation of the very well poised

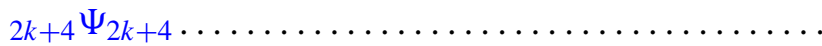

Robert Olin and James E. Thomson, Irreducible operators whose spectra are spectral sets...

Robert John Piacenza, Cohomology of diagrams and equivariant singular

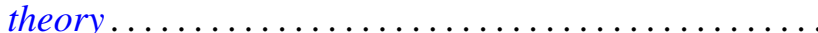

Louis Jackson Ratliff, Jr., Integrally closed ideals and asymptotic prime divisors

Robert Breckenridge Warfield, Jr., Cancellation of modules and groups and stable range of endomorphism rings.................

B. J. Day, Correction to: "Locale geometry" ...............

Stanley Stephen Page, Correction to: "Regular FPF rings" ... 